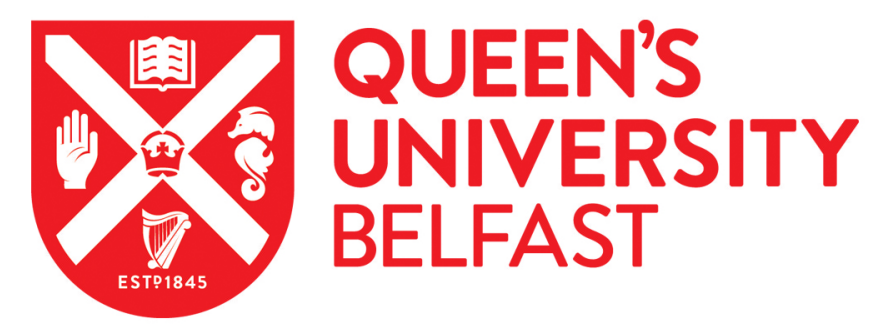

\title{
Microarray patches: potentially useful delivery systems for long-acting nanosuspensions
}

Donnelly, R. F., \& Larrañeta, E. (2017). Microarray patches: potentially useful delivery systems for long-acting nanosuspensions. Drug Discovery Today. https://doi.org/10.1016/j.drudis.2017.10.013

\author{
Published in: \\ Drug Discovery Today \\ Document Version: \\ Peer reviewed version
}

Queen's University Belfast - Research Portal:

Link to publication record in Queen's University Belfast Research Portal

\section{Publisher rights}

This manuscript version is made available under the CC-BY-NC-ND 4.0 license http://creativecommons.org/licenses/by-nc-nd/4.0/,which permits distribution and reproduction for noncommercial purposes, provided the author and source are cited.

\section{General rights}

Copyright for the publications made accessible via the Queen's University Belfast Research Portal is retained by the author(s) and / or other copyright owners and it is a condition of accessing these publications that users recognise and abide by the legal requirements associated with these rights.

Take down policy

The Research Portal is Queen's institutional repository that provides access to Queen's research output. Every effort has been made to ensure that content in the Research Portal does not infringe any person's rights, or applicable UK laws. If you discover content in the Research Portal that you believe breaches copyright or violates any law, please contact openaccess@qub.ac.uk. 


\section{Accepted Manuscript}

Title: Microarray patches: potentially useful delivery systems for long-acting nanosuspensions

Authors: Ryan F. Donnelly, Eneko Larrañeta

PII: S1359-6446(17)30233-7

DOI: https://doi.org/10.1016/j.drudis.2017.10.013

Reference: DRUDIS 2108

To appear in:

Please cite this article as: Donnelly, Ryan F., Larrañeta, Eneko, Microarray patches: potentially useful delivery systems for long-acting nanosuspensions.Drug Discovery Today https://doi.org/10.1016/j.drudis.2017.10.013

This is a PDF file of an unedited manuscript that has been accepted for publication. As a service to our customers we are providing this early version of the manuscript. The manuscript will undergo copyediting, typesetting, and review of the resulting proof before it is published in its final form. Please note that during the production process errors may be discovered which could affect the content, and all legal disclaimers that apply to the journal pertain. 


\title{
Microarray patches: potentially useful delivery systems for long- acting nanosuspensions
}

\author{
Ryan F. Donnelly ${ }^{1}$ and Eneko Larrañeta ${ }^{1}$ \\ 'School of Pharmacy, Queen's University Belfast, Medical Biology Centre, 97 Lisburn Road, Belfast, BT9 7BL, UK
}

Corresponding author: Donnelly, R.F. (r.donnelly@qub.ac.uk)

\section{Highlights}

- Discusses different microneedle types

- Outlines the utility of solid drug nanosuspensions in long-acting drug delivery

- Describes the factors influencing combination of these delivery systems

- Reviews key articles in the field

Keywords: long-acting drug nanosuspensions; microneedles; nanoparticles.

\begin{abstract}
Long-acting drug nanosuspension formulations are coming to the fore as controlled release strategies for several medical conditions and as a preventative measure against HIV infection. However, such delivery systems must, by necessity, be given by hypodermic injection, typically into muscle. This poses problems for patients who are needlephobic, given that injections have to be administered on a weekly or monthly basis. Needle-stick injuries, inappropriate reuse of needles, and poor disposal practices are major challenges in developing countries. Dissolving microneedles (MNs) are capable of delivering high drug doses, if suitably designed and formulated, and are also capable of delivering nanoparticles (NPs) into viable skin. Given that such microneedles are minimally invasive and self-disabling, the potential for major enhancement in patient care and compliance exists. In this review, we explore the key considerations in the development of these combination drug delivery systems.
\end{abstract}

\section{Introduction}

MNs are minimally invasive devices that by-pass the stratum corneum ( $S C$ ) barrier of the skin, thus granting access to the dermal microcirculation and antigen-presenting XXXXX located in the inferior layers of the skin. MNs comprise multiple micron-scale projections positioned on a baseplate in various geometries. When applied to the skin, they painlessly puncture the SC, creating microscopic aqueous channels through which drugs can diffuse. MNs are long enough to penetrate the SC (50-900 Mm in height, up to $2000 \mathrm{MN} \mathrm{cm}^{-2}$ ), but short enough to avoid stimulation of dermal nerves. They are manufactured from various materials (e.g., silicon, metal, or polymer using microfabrication techniques [1-3]. Originally described in a 1970s patent and finally realized in practical terms during the late 1990s [4], MNs (Figure 1) are currently of great interest because of several advantages that they have over traditional methods of drug delivery. Some of these advantages include the ability to painlessly administer the active pharmaceutical ingredient (API), bypass the hepatic first-pass metabolism, and the extension of the variety of drug types that can be delivered both intra- and transdermally.

The first substance delivered using MNs was the low-molecular-weight compound, calcein [4]. Multiple investigations rapidly followed, leading to the current ever-growing body of evidence for the significant drug delivery capabilities of MNs. Although a variety of strategies has been used (Figure 2), MNs fabricated from silicon and metal continue to be extensively investigated for drug delivery. Their use typically involves the pretreatment of skin, followed by application of a topical solution, gel, or patch containing the drug to be delivered [5-7]. Although this conventional 'poke and patch' methodology has progressed somewhat from the original studies, it has been recognized that such a cumbersome two-step application process is a major drawback [8].

To create a one-step application process, solid MNs have been coated with the material to be delivered. Coated MNs have been used for the delivery of several different compounds, including fluorescein sodium [9], salmon calcitonin [10], desmopressin [11], parathyroid hormone (PTH) [12], and DNA/RNA [13,14], among others. Aside 
from this, because of the limited drug-loading capacity of this method, coated MNs are more frequently used for the delivery of highly potent molecules and vaccines.

Research into hollow MNs has focused mainly on array design and characterization, with several sophisticated engineering strategies presented [15-18]. However, a major limitation to their use is the potential blockage of the MN bore by compressed dermal tissue upon insertion, reducing drug release [18].

Dissolving MNs have been used to deliver several small-molecule drugs, including caffeine, lidocaine, theophylline, and metronidazole [19]. Additionally, they have been used to specifically target various clinical needs by the delivery of several biopharmaceutical molecules, including low-molecular-weight heparins [20], insulin [21], leuprolide acetate [22], erythropoietin [23], and human growth hormone [24]. A central criticism of the dissolving platform was the perceived inability to deliver therapeutically relevant doses of low-potency drug substances [25]. However, McCrudden et al. have taken steps to address these concerns, having successfully delivered therapeutically relevant doses of ibuprofen sodium in a rat model [26].

Hydrogel-forming $\mathrm{MNs}$ have been demonstrated to proficiently deliver both small molecules, such as metronidazole and theophylline, and larger molecules, such as insulin and proteins [8]. The benefit of the hydrogel system is that the MN swelling rate can be controlled by altering the polymer cross-linked density, thus conferring the ability to govern drug release rate, which can be tailored for specific drugs. The delivered dose is not limited by what can be loaded into the needles themselves, given that the drug is contained within an attached solid drug reservoir. Accordingly, sustained delivery of high drug doses is readily achievable.

As can be seen from Table 1, there has been a range of completed and ongoing clinical trials involving the use of MNs. Although several investigations involving humans have considered the perception, safety, and practical applications of MN technology [27,28] and a few human volunteer trials have studied MN-mediated transdermal drug delivery [12,29], the predominant focus in the field to date has been on vaccines [30-33]. This is hardly surprising, given the potential for a stable, dry-state formulation, the avoidance of needle-stick injuries common with hypodermic syringes, dose-sparing through direct targeting of the abundance of professional antigenpresenting cells in viable skin, and the self-disabling nature of dissolving MNs. Consequently, several clinical trials covering the use of MNs for vaccine delivery are detailed in Table 1. Influenza intradermal vaccination has been extensively studied because there is a constant demand for a seasonal vaccine. These trials were conducted around the world in thousands of volunteers, including a randomized, open-label Phase 2 clinical trial (978 healthy adults) [34], a Phase 3 randomized, double-blind trial (2255 healthy adults) [35], and a Phase 2/3 trial in older individuals (aged 60 years and older) [36]. The obtained results suggested that the MN-based vaccine provided an equivalent (and, in some cases, superior) immune response compared with the conventional intramuscular vaccine.

$\mathrm{MN}$ vaccines have the potential to revolutionize vaccination, especially in the developing world. However, in studies where the delivery of therapeutic drug substances using MNs has been exemplified, the focus has tended to be on illustration of the capability of MNs to deliver a substance with particular physicochemical characteristics and little mention is typically made of the actual amount delivered or its relevance to therapeutic human doses.

Vaccines tend to be potent and, thus, delivery of even microgram quantities of antigen, antigen/adjuvant combination, virus-like particle, or even DNA is often sufficient to elicit an immune response, especially when targeted to the viable epidermis and/or dermis. This means that small, postage stamp-sized MN patches that can be inserted into the skin by fairly gentle thumb pressure are sufficient to achieve successful vaccination.

In addition to MN-mediated vaccination, the delivery of insulin using MNs has been extensively studied in clinical trials (Table 1). The use of $\mathrm{MNs}$ for this purpose provided better patient compliance compared with traditional subcutaneous injections. In addition, clinical trials showed that the MN-mediated administration of insulin enabled faster uptake of this molecule and equivalent bioavailability/blood-glucose effects compared with subcutaneous administrations [37,38].

The use of MN devices for clinically relevant does in human volunteers has not been extensively studied, as shown in Table 1. However, recent work in the field has focused strongly on the transdermal delivery of therapeutically relevant doses of drugs using MN patches. Plasma levels in animal models have been measured and extrapolated to estimate suitable patch sizes for the achievement of therapeutic plasma levels in humans $[18,20,22]$. Given that most commonly used small-molecule drugs tend to require oral doses in the range of tens to hundreds of milligrams daily, the patch sizes estimated have ranged from $10 \mathrm{~cm}^{2}$ to $30 \mathrm{~cm}^{2}$. Such patch sizes are well within the range of marketed transdermal patches. Indeed, Novartis market Nicotinell ${ }^{\circledR}$ (nicotine) patches of $30 \mathrm{~cm}^{2}$ (www.nicotinell.co.uk), whereas Janssen market Duragesic ${ }^{\circledR}$ CII (fentanyl) patches of 32 and $42 \mathrm{~cm}^{2}$ (www.duragesic.com). It was recently shown that human volunteers can insert the MNs of large patches as efficiently and reliably as those on smaller patches, thus making such a delivery system viable [39].

The ability to deliver high drug doses has raised interest in the possibility of delivering long-acting drug nanosuspensions intradermally without the need for a conventional hypodermic needle injection. Nanosuspensions are, in simple terms, aqueous suspensions of NPs made from solid-drug crystals stabilized with a coating of surfactant and/or polymer. They are typically up to $90 \% \mathrm{w} / \mathrm{w}$ pure drug and can be prepared by 'top-down' (e.g., wet milling or high-pressure homogenization) or 'bottom-up' (solution-based nanoprecipitation) methods [40-42]. Preparation of solid-drug NPs can be used to improve aqueous solubility for applications in enhanced oral or pulmonary bioavailability. However, if one selects a potent drug with relatively poor aqueous solubility and can tailor particle size appropriately, an injectable preparation capable of sustained delivery of clinically relevant drug doses for up to 3 months from a single injection can be produced. The particles are deposited as a depot and slowly 
release drug for absorption into the systemic circulation as they dissolve in interstitial fluid. If enough particles can be deposited, then therapeutic plasma levels can be maintained for prolonged periods [40-42].

Originally used for the hormonal treatment of endometriosis, long-acting drug nanosuspensions then found use in the management of schizophrenia. Most interestingly, however, this formulation type is now undergoing clinical trials for the prevention and treatment of HIV [42]. It is because of this exciting new indication that the mode of nanosuspension delivery has come sharply into focus. Oral administration of nanosuspensions does not allow sustained drug absorption over weeks or months and, thus, such products have typically been administered subcutaneously or, if higher volumes are required (up to $2.7 \mathrm{ml}$ from a single injection) to deliver therapeutic doses, intramuscularly. Hypodermic needle injections cause problems in developing countries, in particular, because of the lack of skilled healthcare personnel, frequency of needle-stick injuries, inappropriate reuse of needles, and poor disposal practices. An alternative, minimally invasive, self-disabling, delivery system that avoids such problems would not only be particularly useful in low-resource settings, but could also improve compliance in developed countries.

Solid MN arrays have been used to enhance the delivery of microparticle and NP suspensions. However, in these cases, the application process requires the application of a solid MN array before administration of a formulation containing the suspension into the treated area [17,43,44]. A two-step process is not ideal from a patient point of view.

Several examples of hollow MNs for the delivery of nanosuspensions can be found in the literature [45-48]. These devices have been extensively studied for the intraocular administration of NPs and microparticles [46-48]. However, they require complex pumping systems, which are expensive and difficult to manufacture and could present issues for correct use in the absence of skilled healthcare workers in developing countries [2]. The safe and hygienic disposal of such systems in low-resource settings might also prove problematic. Coated MNs have limited dosing capacity and are also removed from the skin intact, therefore presenting challenges for disposal [2]. Diffusion of hydrophobic NPs through swollen hydrogel matrices is likely to be poor, thus ruling that system out [8]. However, dissolving $\mathrm{MNs}$ have considerable promise in the intradermal delivery of long-acting nanosuspensions.

The literature contains a variety of examples of the use of dissolving MNs for nanosuspension administration [49-51]. However, most of these papers focused on the delivery of encapsulated vaccines [49,52,53] or were designed as a proof of concept to show the capabilities of MNs to enhance the delivery of particulate formulations through the SC [49]. Consequently, basic technical and scientific challenges are being addressed currently, such as: the possibility of manufacturing two-layered MN arrays concentrating suspensions in the needle tips or the study [54,55] of the fate of NPs administered intradermally [55]. Nevertheless, the delivery of therapeutically relevant doses of nanomedicines using dissolving MNs remains relatively unexplored.

Advantages of a dissolving MN system over conventional needle-and-syringe-based methods for the administration of long-acting drug nanosuspensions would include: (i) no requirement for skilled medical personnel to administer the dose; (ii) potential for at-home use by patients; (iii) possibility for improved compliance; (iv) avoidance of needle-stick injuries; (v) specialized disposal not required; and (vi) possibility for enhanced storage stability because of the dried nature of the formulations.

However, to be a realistic proposition, such a dissolving MN system would need to be able to incorporate a high loading of hydrophobic NPs in the needles themselves. Given the viscous nature of the gel formed in skin upon needle dissolution, it is unlikely that any NPs in the baseplate upon which the needles were formed would be able to diffuse into the viable skin. Doses of several hundred milligrams would, in most cases (apart from hormones), have to be delivered into skin upon needle dissolution. If the patch size required to deliver such a dose would, by necessity, be greater than that of conventional transdermal patches, then the delivery of a particular drug would not be feasible. However, if one considers that the MNs themselves can weigh $10 \mathrm{mg}$ for every $\mathrm{cm}^{2}$ of patch upon which they are formed and the drug loading is a minimum of $80 \% \mathrm{w} / \mathrm{w}$, then $8 \mathrm{mg} / \mathrm{cm}^{2}$ of drug could be delivered upon total needle dissolution in skin. If the drug dose for a month of treatment were $300 \mathrm{mg}$, then the patch size would be $37.5 \mathrm{~cm}^{2}$. If the drug dose were $600 \mathrm{mg}$, then two patches could be applied, one on each arm. This mimics the current rilpivirine regimen for HIV prevention, where two injections are given at different sites. Given that large MN patches can be reliably applied by human volunteers, the approach appears feasible. However, what would be useful would be an indicator confirming when the patch can be removed. A low-cost system would not be able to tell when the needles had dissolved, but a moisture sensor or time-dependent color change indicator based on average in-skin dissolution time would not add prohibitive expense.

The needles should dissolve quickly in skin (in $<1 \mathrm{~h}$ ideally), so as to be convenient for patients. This means that the polymer matrix must be carefully chosen so as to provide sufficient mechanical strength to allow ready puncture of the SC upon application of relatively gentle pressure, while not being brittle, but must not be slowly soluble in the relatively small volume of fluid available in viable skin. Given that possibly $>80 \%$ by weight of the MN might comprise hydrophobic NPs, repulsion of interstitial fluid could be a consideration requiring addition of disintegrants to boost MN breakdown. Excipients chosen, including the matrix polymer and water, must not significantly alter the characteristics of the particles of the nanosuspension (e.g., size, charge or aggregation status) or cause dissolution during manufacture or storage. The excipients should also be biodegradable or of sufficiently low molecular weight to allow ready clearance from the body, given that the patch will need to be applied by the patient weekly or monthly. Accumulation of excipients in skin or elsewhere in the body would be undesirable and 
would be likely to raise regulatory concerns. Indeed, from a regulatory viewpoint, clinical studies aimed at supporting market authorization would need to show that therapeutic blood levels comparative to existing dosage forms are achievable, even if the pharmacokinetic patterns differ. Whether regulators would require a sterile or low bioburden product, considering that MNs enter viable skin, is not clear as yet. It is likely that a suitable applicator, or an in-built method of confirming correct use (MN insertion) would also be required, especially if the product is to be used by patients themselves in low-resource settings. In-built feedback mechanisms would be more desirable than applicator devices, especially if the cost could be kept low. Such systems have recently been described [39,56,57].

Dissolving MN systems loaded with hydrophobic NPs are likely to be manufactured in two stages to avoid waste of drug (Figure 3a). The baseplate could be separately produced as a polymeric film using well-established knifecasting techniques. Mixing a freeze-dried NP powder with a polymeric gel or adding polymer powder directly to a concentrated nanosuspension might be required to maximize drug loading in the needles of the array. A suitable mold could be filled by the particle-loaded gel by a range of methods, including applying a vacuum or utilising compressed air. Needles and baseplate can then be merged before or after drying, using a thin adhesive layer, if necessary. For most drugs, the NPs are likely to have to be distributed throughout the entire length of the needles (Figure 3b), which could be increased to further enhance the drug-loading capacity, while taking care not to exceed $1 \mathrm{~mm}$, in which case pain and pinprick bleeding can occur. However, for potent hormones, the MN could be formed by two gel castings so as to localize the drug in the needle tips alone (Figure 3c).

Some of the manufacturing approaches previously described have been evaluated by different research groups for the laboratory-scale preparation of MNs. However, one of the important challenges in MN technology is the scale-up the manufacturing processes. Several studies described new processes to improve the manufacturing of MN arrays [58,59]. Lutton et al. described a scalable method for MN production using a micromolding procedure [58]. The described method can be easily applied to the manufacturing of nanosuspension-containing MN arrays. Nevertheless, there is another key issue that needs to be addressed to design a suitable manufacturing process: product sterility/low bioburden requirements [60]. As described previously, the regulatory bodies have not yet defined the requirements for MN products in terms of sterility. Consequently, the manufacturing procedures should be designed with this challenge in mind.

\section{Concluding remarks}

Dissolving MN delivery systems have been shown to deliver NPs into the viable skin layers in vitro and in vivo [55] and have the capability to incorporate and release high doses of undissolved solids [26]. Accordingly, their potential as a next-generation delivery system for emerging long-acting nanosuspensions is becoming clear. Indeed, the first report of the development and successful in vivo evaluation of such a combination system was recently presented [61]. To date, MNs, including dissolving MNs, have been rather narrowly viewed as vaccine delivery systems for the developing world. However, the demonstrated ability of properly formulated systems to incorporate and release high drug doses should soon begin to change this mindset. One could view the dissolving MN simply as a tool to deposit the 'real' delivery system, the drug nanosuspension, in the viable skin layers in sufficient amounts to allow prolonged drug administration. It is likely that we will soon see an increasing number of hydrophobic solid drug nanoparticulate systems delivered using dissolving MNs, with a variety of therapeutic indications. For translation to clinic and, ultimately market, methods of manufacture will need to be refined and scaled up [62] and the 'microneedle' aspect of the name of the final patch systems removed. The term currently being used by the World Health Organization for all microneedle-based systems is 'microarray patches', or MAPs. Such an adjustment might appear minor, but could be important for patient acceptance. Dissolving MAPs could well be the dosage form of the near future, with needle-free administration of long-acting drug nanosuspensions an exciting application with a range of potential benefits for patients and healthcare providers, especially in the poorest countries of the world. Watch this space! 


\section{References}

1 Arora, A. et al. (2008) Micro-scale devices for transdermal drug delivery. Int. J. Pharm. 364, 227-236

2 Donnelly, R.F. et al. (2012) Microneedle-Mediated Transdermal and Intradermal Drug Delivery, Wiley

3 Tuan-Mahmood, T. et al. (2013) Microneedles for intradermal and transdermal drug delivery. Eur. J. Pharm. Sci. 50, 623-637

4 Henry, S. et al. (1998) Microfabricated microneedles: a novel approach to transdermal drug delivery. J. Pharm. Sci. 87, 922-925

5 Zhou, C.P. et al. (2010) Transdermal delivery of insulin using microneedle rollers in vivo. Int. J. Pharm. 392127-133

6 Mikolajewska, P. et al. (2010) Microneedle pre-treatment of human skin improves 5-aminolevulininc acid (ALA)- and 5-aminolevulinic acid methyl ester (MAL)-induced PpIX production for topical photodynamic therapy without increase in pain or erythema. Pharm. Res. 27, $2213-2220$

7 Stahl, J. et al. (2012) Microneedle pre-treatment enhances the percutaneous permeation of hydrophilic compounds with high melting points. BMC Pharmacol. Toxicol. 13, 5

8 Donnelly, R.F.et al. (2012) Hydrogel-forming microneedle arrays for enhanced transdermal drug delivery. Adv. Funct. Mater. 22, 4879-4890

9. Chen, J. et al. (2013) Controllable coating of microneedles for transdermal drug delivery. Drug Dev. Ind. Pharm. 41, 415-422

10 Tas, C. et al. (2012) Delivery of salmon calcitonin using a microneedle patch. Int. J. Pharm. 423, 257-263

11 Cormier, M. et al. (2004) Transdermal delivery of desmopressin using a coated microneedle array patch system. J. Control. Release 97, 503511

12 Daddona, P.E. et al. (2011) Parathyroid hormone (1-34)-coated microneedle patch system: clinical pharmacokinetics and pharmacodynamics for treatment of osteoporosis. Pharm. Res. 28, 159-165

13 Pearton, M. et al. (2012) Microneedle delivery of plasmid DNA to living human skin: formulation coating, skin insertion and gene expression. J. Control. Release 160, 561-569

14 Chong, R.H.E. et al. (2013) Gene silencing following siRNA delivery to skin via coated steel microneedles: in vitro and in vivo proof-ofconcept. J. Control. Release 166, 211-219

15 Wang P.C. et al. (2014) Hypodermic-needle-like hollow polymer microneedle array: fabrication and characterization. J. Micromechan. Syst. 23, 991-998

16 Martanto, W. et al. (2006) Microinfusion using hollow microneedles. Pharm. Res. 23, 104-113

17 McAllister, D.V. et al. (2003) Microfabricated needles for transdermal delivery of macromolecules and nanoparticles: fabrication methods and transport studies, Proc. Natl. Acad. Sci. U. S. A. 100, 13755-13760

18 Gardeniers, H.J.G.E. et al. (2003) Silicon micromachined hollow microneedles for transdermal liquid transport. J. Micromechan. Syst. 12 , 855-862

19 Garland, M.J. et al. (2012) Influence of skin model on in vitro performance of drug-loaded soluble microneedle arrays. Int. J. Pharm. 434, $80-89$

20 Gomaa, Y.A. et al. (2012) Laser engineered dissolving microneedles for active transdermal delivery of nadroparin calcium. Int. J. Pharm. $82,299-307$

21 Migalska, K. et al. (2011) Laser-engineered dissolving microneedle arrays for transdermal macromolecular drug delivery. Pharm. Res. 28, 1919-1930

22 Ito, Y. et al. (2011) Incidence of low bioavailability of leuprolide acetate after percutaneous administration to rats by dissolving microneedles. Int. J. Pharm. 407, 126-131

23 Ito, Y. et al., (2010) Self-dissolving micropile array chip as percutaneous delivery system of protein drug. Biol. Pharm. Bull. 33, 683-690

24 Lee, J.W. et al. (2011) Dissolving microneedle patch for transdermal delivery of human growth hormone. Small 7, 531-539

25 Tuan-Mahmood, T.M. et al. (2013) Microneedles for intradermal and transdermal drug delivery. Eur. J. Pharm. Sci. 44, 623-637

26 McCrudden, M.T.C. et al. (2014) Design and physicochemical characterisation of novel dissolving polymeric microneedle arrays for transdermal delivery of high dose, low molecular weight drugs. J. Control. Release 180C, 71-80

27 Bal, S.M. et al., (2008) In vivo assessment of safety of microneedle arrays in human skin. Eur. J. Pharm. Sci. 35, 193-202

28 Donnelly, R.F. et al. (2014) Hydrogel-forming microneedle arrays can be effectively inserted in skin by self-application: a pilot study centred on pharmacist intervention and a patient information leaflet. Pharmacol. Res. 31, 1989-1999

29 Wermeling, D.P. et al. (2008) Microneedles permit transdermal delivery of a skin-impermeant medication to humans. Proc. Natl. Acad. Sci. U. S. A. 105, 2058-2063

30 McGrath, M.G. et al. (2011) Determination of parameters for successful spray coating of silicon microneedle arrays. Int. J. Pharm. 415, 140149

31 Yang, H.W. et al. (2017) Ebola vaccination using a DNA vaccine coated on PLGA-PLL/ $\gamma$ PGA nanoparticles administered using a microneedle patch. Adv. Healthc. Mater. 6, 1600750

32 Pearton, M. et al. (2013) Host responses in human skin after conventional intradermal injection or microneedle administration of virus-likeparticle influenza vaccine. Adv. Healthc. Mater. 2, 1401-1410

33 Fernando, G.J. et al. (2016) Influenza nucleoprotein DNA vaccination by a skin targeted, dry coated, densely packed microprojection array (Nanopatch) induces potent antibody and CD8(+) T cell responses. J. Control. Release 237, 35-41

34 Leroux-Roels, I. et al. (2008) Seasonal influenza vaccine delivered by intradermal microinjection: aA randomised controlled safety and immunogenicity trial in adults. Vaccine 26, 6614-6619

35 Arnou, R. et al. (2010) Immunogenicity, large scale safety and lot consistency of an intradermal influenza vaccine in adults aged 18-60 years: randomized, controlled, Phase III trial. Hum. Vaccines 6, 346-354

36 Holland, D. et al. (2008) Intradermal influenza vaccine administered using a new microinjection system produces superior immunogenicity in elderly adults: a randomized controlled trial. J. Infect. Dis. 198, 650-658

37 Gupta, J. et al. (2009) Minimally invasive insulin delivery in subjects with type 1 diabetes using hollow microneedles. Diabetes Technol. Ther. 11, 329-337

38 Gupta, J. et al. (2011) Rapid pharmacokinetics of intradermal insulin administered using microneedles in type 1 diabetes subjects, Diabetes Technol. Ther. 13, 451-456

39 Ripolin, A. et al. (2017) Successful application of large microneedle patches by human volunteers. Int. J. Pharm. 521, 92-101

40 Sun, B. et al. (2012) Nanocrystals for the parenteral delivery of poorly water-soluble drugs. Curr. Opin. Solid State Mater. Sci. 16, 295-301

41 Chin, W.W. et al. (2014) A brief literature and patent review of nanosuspensions to a final drug product. J. Pharm. Sci. 103, 2980-2999

42 Own, A. and Ranard, S. (2016) Strengths, weaknesses, opportunities and challenges for long acting injectable therapies: Insights for applications in HIV therapy. Adv. Drug Deliv. Rev. 103,144-156

43 Gomaa, Y.A. (2014) Microneedle/nanoencapsulation-mediated transdermal delivery: mechanistic insights. Eur. J. Pharm. Biopharm. 86, $145-155$

44 Wing, S.C. (2015) Drug delivery into microneedle-porated nails from nanoparticle reservoirs. J. Control. Release 220. 98-106

45 Häfeli, U.O. et al. (2009) In vivo evaluation of a microneedle-based miniature syringe for intradermal drug delivery. Biomed. Microdevices 11, 943-950 
46 Kim, Y.C. et al. (2014) Particle-stabilized emulsion droplets for gravity-mediated targeting in the posterior segment of the eye. Adv. Healthc. Mater. 3, 1272-1282

47 Patel, S.R. et al. (2011) Suprachoroidal drug delivery to the back of the eye using hollow microneedles. Pharm. Res. 28, 166-176

48 Jiang, J. et al. (2009) Intrascleral drug delivery to the eye using hollow microneedles. Pharm. Res. 26, 395-403

49 Larrañeta, E. et al. (2016) Microneedles: a new frontier in nanomedicine delivery. Pharm. Res. 33, 1055-1073

50 Ke, C.J. et al. (2012) Multidrug release based on microneedle arrays filled with pH-responsive PLGA hollow microspheres. Biomaterials 33, $5156-5165$

51 Lee, S.G. et al. (2014) Nanostructured lipid carrier-loaded hyaluronic acid microneedles for controlled dermal delivery of a lipophilic molecule. Int. J. Nanomedicine 9, 289-299

52 Cole, G. et al. (2017) Dissolving microneedles for DNA vaccination: improving functionality via polymer characterization and RALA complexation. Hum. Vaccin. Immunother. 13, 50-62

53 Yang, H.W. et al. (2017) Ebola vaccination using a DNA vaccine coated on PLGA-PLL/gamma PGA nanoparticles administered using a microneedle patch. Adv. Healthc. Mater. 6, 1600750

54 Lalit, V. and Donnelly, R.F. (2017) Novel bilayer dissolving microneedle arrays with concentrated PLGA nano-microparticles for targeted intradermal delivery: proof of concept. J. Control. Release. Published online October 14 , 2017. http://www.doi.org/10.1016/j.jconrel.2017.10.005

55 Kennedy, J. et al. (2017) In vivo studies investigating biodistribution of nanoparticle-encapsulated rhodamine B delivered via dissolving microneedles. J. Control. Release. Published online April 14, 2017. http://www.doi.org/10.1016/j.jconrel.2017.04.022

56 Vicente-Pérez, E.M. et al. (2016) The use of a pressure-indicating sensor film to provide feedback upon hydrogel-forming microneedle array self-application in vivo. Pharm. Res. 33, 3072-3080

57 Norman, J.J. et al. (2014) Microneedle patches: usability and acceptability for self-vaccination against influenza. Vaccine 32, 1856 -1862

58 Lutton, R.E.M. et al. (2015) A novel scalable manufacturing process for the production of hydrogel-forming microneedle arrays. Int. J. Pharm. 494, 417-429

59 Larrañeta, E. et al. (2015) Microwave-assisted preparation of hydrogel-forming microneedle arrays for transdermal drug delivery applications. Macromol. Mater. Eng. 300, 586-595

60 Lutton, R.E.M. et al. (2015) Microneedle characterisation: the need for universal acceptance criteria and GMP specifications when moving towards commercialisation. Drug Deliv. Transl. Res. 5, 313-331

61 McCrudden, M.T.C. et al. (2016) Two-step casting of dissolving MN arrays for use in the sustained release of rilpivirine for HIV preexposure prophylaxis. Proc. 43rd Annu. Meet. Controlled Release Soc. 2016, 174

62 Larraneta, E. et al. (2016) Microneedle arrays as transdermal and intradermal drug delivery systems: materials science, manufacture and commercial development. Mater. Sci. Eng. R 104, 1-32 
Figure 1. Microneedle (MN) designs. Wet-etched silicon $\mathrm{MNs}$ approximately $280 \mathrm{Mm}$ in height suitable for coating with capture proteins or antibodies (a,b). MNs approximately $600 \mathrm{Mm}$ in height produced from micromolding of aqueous gels of poly(methylvinylether-co-maleic acid) and poly(ethylene glycol) (PEG) 10 000 that swell in skin to capture skin interstitial fluid (c). Poly(carbonate) MNs approximately $1000 \mathrm{Mm}$ in height with a $100 \mathrm{Mm}$ off-center through-hole suitable for blood extraction (d), Orion Helium-ion microscope images of 7-MN arrays of this design (e) and 3D optical coherence tomographic representation of these MNs in situ, following insertion into excised neonatal porcine skin in vitro (f). Swollen hydrogel-forming MNs approximately $600 \mathrm{Mm}$ in height produced from micromolding of aqueous gels of poly(methylvinylether-co-maleic acid) and PEG 10000 completely intact following removal from skin (g) and MNs approximately $280 \mathrm{Mm}$ in height produced from micromolding of aqueous gels of poly(methylvinylether-co-maleic acid) and glycerol following removal from skin (h). The latter type of hydrogel-forming $\mathrm{MNs}$ following uptake of meso-tetra ( $\mathrm{N}$-methyl-4-pyridyl) porphine tetra tosylate in vitro (I). Hydrogel-forming $\mathrm{MNs}$ approximately $600 \mathrm{Mm}$ in height produced from micromolding of aqueous gels of poly(methylvinylether-co-maleic acid) and PEG 10000 swelling in human skin in vivo (j).

Figure 2. Microneedle (MN) delivery strategies. A schematic representation of five different MN types used to facilitate drug delivery transdermally. (a) Solid MNs for increasing the permeability of a drug formulation by creating microholes across the skin. (b) Coated MNs for rapid dissolution of the coated drug into the skin. (c) Dissolvable MNs for rapid or controlled release of the drug incorporated within the MNs. (d) Hollow MNs used to puncture the skin and enable release of a liquid drug following active infusion or diffusion of the formulation through the needle bores. (e) Hydrogel-forming MNs take up interstitial fluids from the tissue, inducing diffusion of the drug located in a patch through the swollen microprojections.

Figure 3. Microarray patches loaded with long-acting nanosuspensions. Preparation of microarray patches using aqueous polymeric gels containing solid-drug nanosuspensions where the preformed baseplate is added after initial casting of the gel into the molds (a). Delivery of solid-drug nanosuspensions into skin where the particles are loaded into the entire shaft length of the microneedles (MN) (b). Delivery of solid-drug nanosuspensions into skin where the particles are loaded into the MN tips only (c).
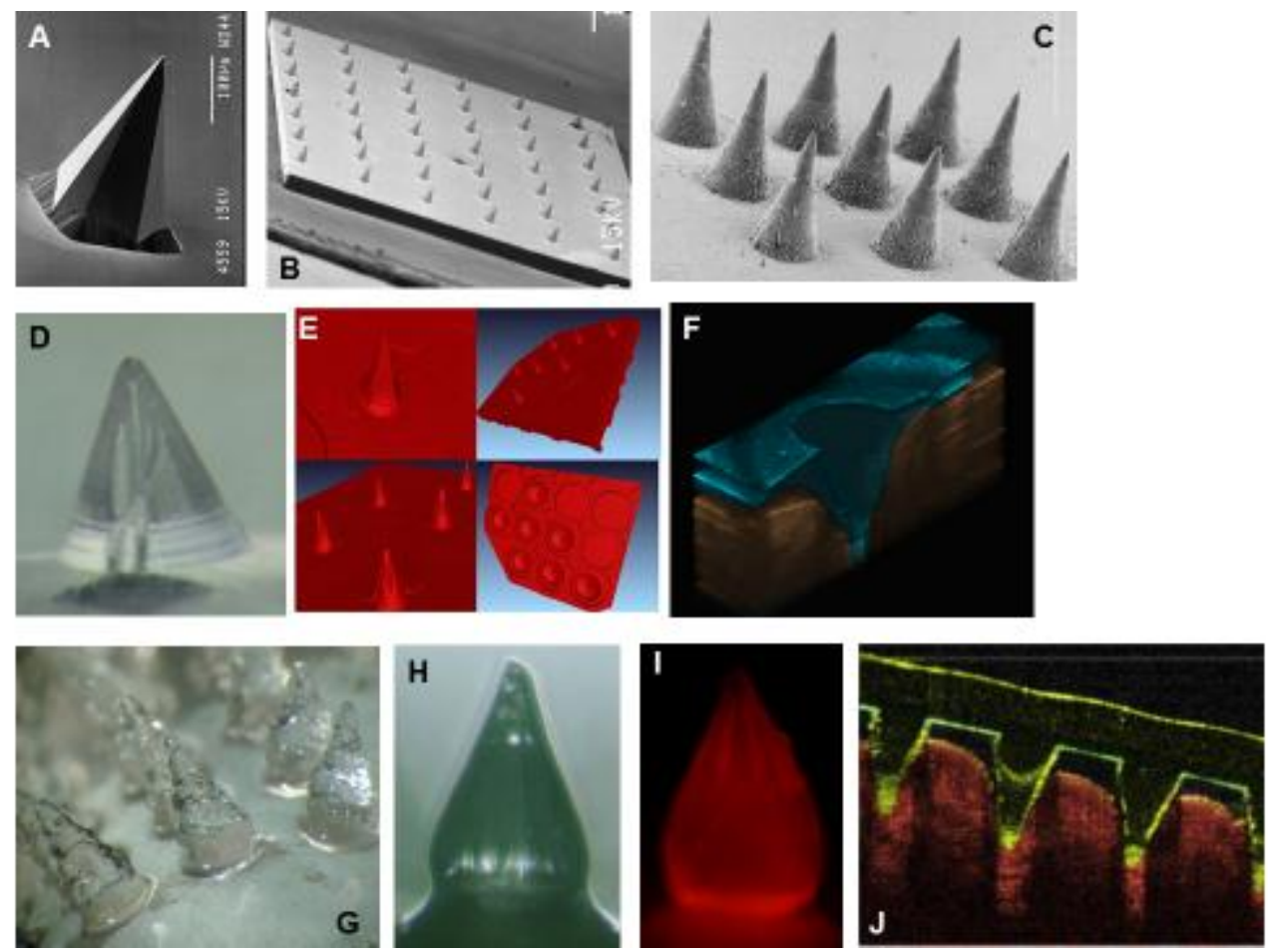

Figure 1 


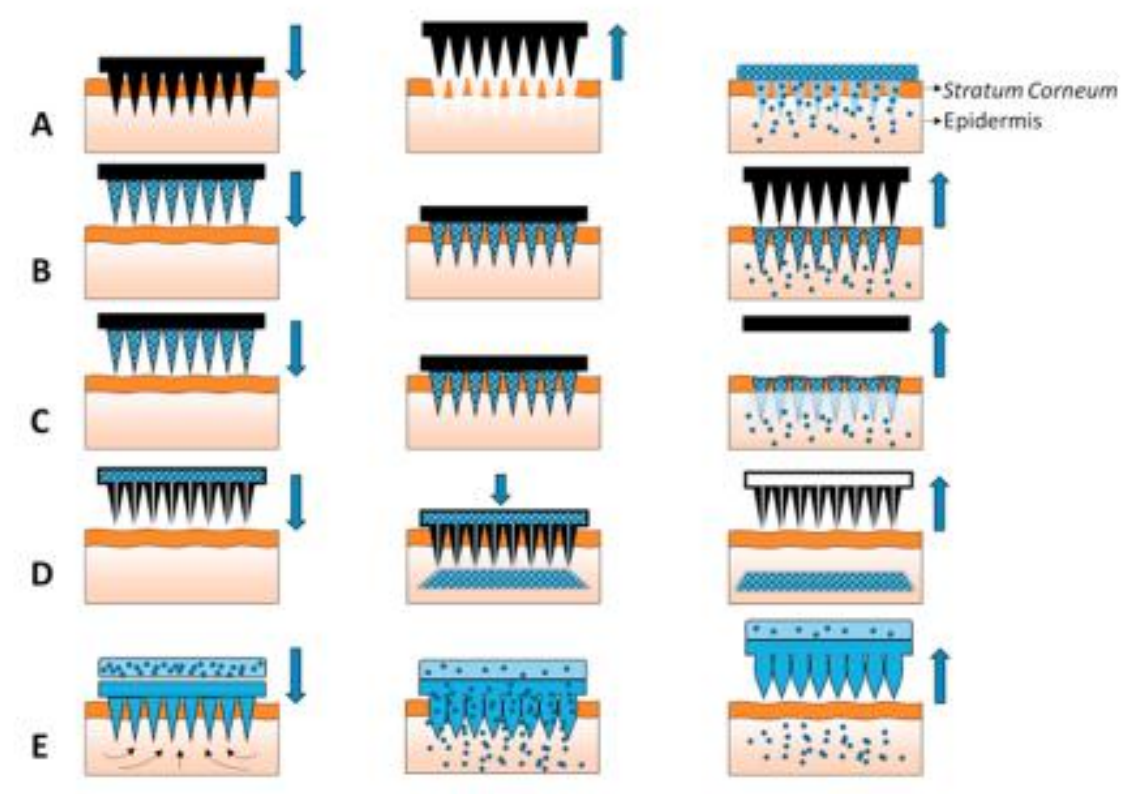

Figure 2

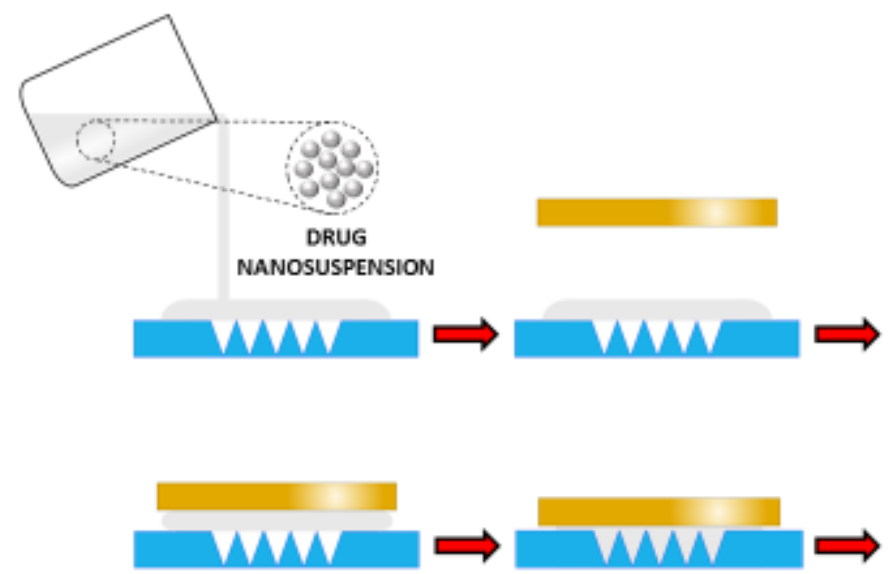

Figure 3A 

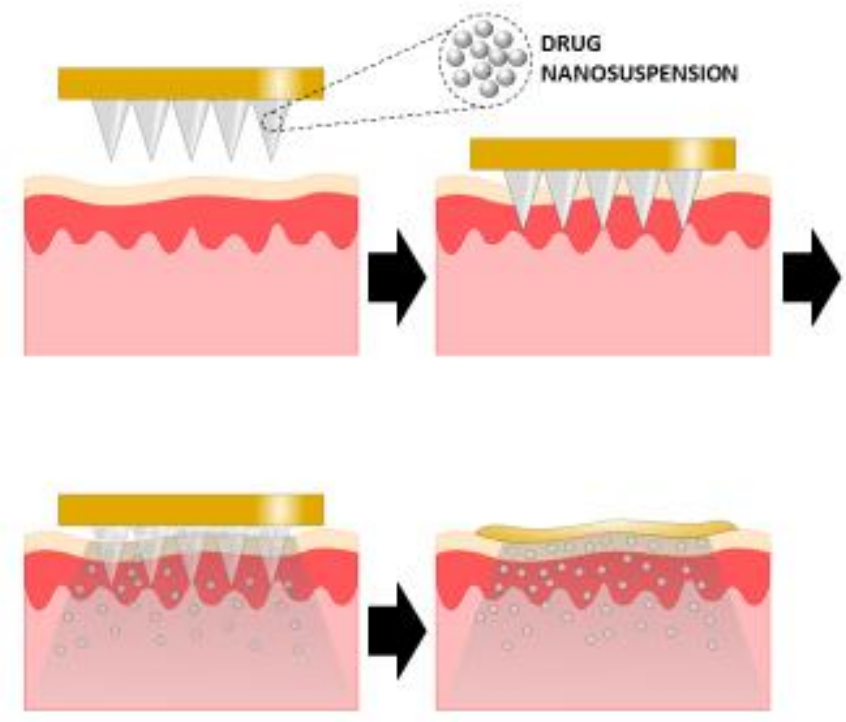

Figure 3B

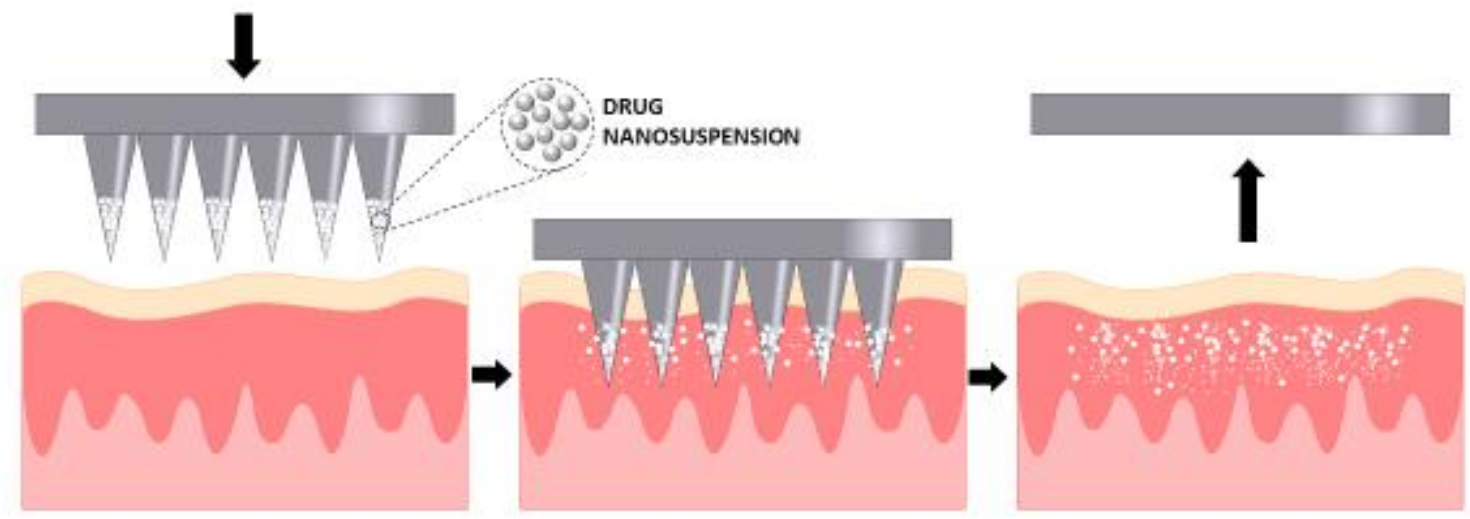




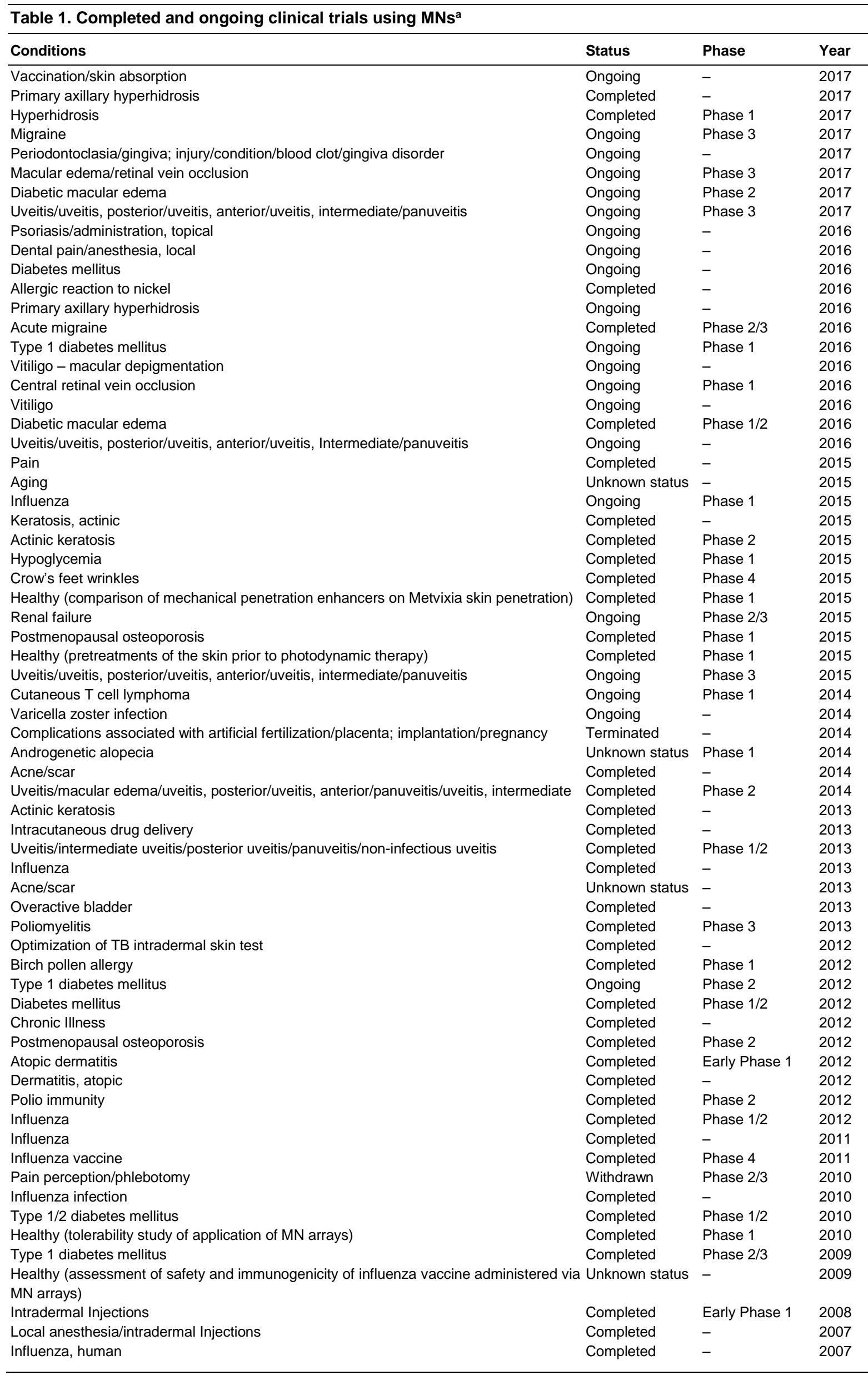


